\title{
Nonlinear Vehicle Velocity Observer with Road-Tire Friction Adaptation
}

\author{
Håvard Fjær Grip ${ }^{\star}$ Lars Imsland Tor A. Johansen` Thor I. Fossen` Jens C. Kalkkuhl Avshalom Suissa \\ SINTEF ICT, Applied Cybernetics \\ NO-7465 Trondheim, Norway \\ DaimlerChrysler Research and Technology \\ 70456 Stuttgart, Germany
}

\begin{abstract}
A nonlinear observer for lateral velocity of an automotive vehicle is extended for robustness with respect to unknown road surface conditions. The observer uses a friction model parametrized with the maximum road-tire friction coefficient, and an adaptive parameter update law is designed for estimation of this coefficient. The adaptive nonlinear observer is proven to be uniformly globally asymptotically stable under a uniform $\delta$-persistency-of-excitation condition, and a set of additional technical assumptions, using results related to Matrosov's Theorem. The adaptive observer is validated using experimental data from a car.
\end{abstract}

\section{INTRODUCTION}

A recent focus of the car industry has been the development of safety systems which actively assist the driver in order to avoid dangerous situations. As such systems become more advanced, they depend to an increasing extent on accurate information about the state of the vehicle and its surroundings. Much —although not all—of this information can be obtained by direct measurement, but the appropriate sensors may be unreliable, inaccurate or prohibitively expensive. Observers are therefore used to provide estimates of important states. Some observers use a road-tire friction model internally, and therefore rely on information about the road surface conditions, usually represented in the friction model by a friction parameter. This situation forms the basis of this paper.

Existing schemes for estimating road surface conditions vary in their approach. In [1], a least-squares method is used on measurements of wheel angular velocity to estimate the slope of the friction force versus tire slip. An observer for lateral velocity in [2] includes a filtering scheme for estimating the maximum road-tire friction coefficient, by primarily using the lateral acceleration measurement during times when this provides a good measurement of the coefficient. A similar approach is taken in [3]. In [4], a Kalman filter is used to classify road surface conditions, by inspecting the ratio between slip values of the driven wheels and the normalized friction force, obtained using wheel angular velocities and engine torque. In [5], an extended Kalman filter (EKF) is combined with statistical methods in order to estimate the maximum road-tire friction coefficient, using measurements of the yaw and roll rates, wheel angular velocities, and longitudinal and lateral accelerations, as well as knowledge of the steering angle and total brake line pressure. Other examples of EKFs are presented in [6] and

This research is supported by the European Commission STREP project CEmACS, contract 004175 .

${ }^{\star}$ Also affiliated with NTNU, Department of Engineering Cybernetics, NO-7491 Trondheim, Norway.
[7]. In [8], wheel angular velocity, longitudinal tire slip and wheel torque is used to generate an estimate of the wheel angular velocity and for adaptation of a friction parameter. Wheel angular velocity and torque is used in [9] for estimation of the longitudinal velocity and wheel angular velocity, and adaptation of a friction parameter. In both [8] and [9], convergence of the adapted friction parameters under conditions of nonzero longitudinal tire slip is studied.

In [10], a numerically efficient, nonlinear observer based on a modular design was presented with stability guarantees. The contribution of this paper is to present a solution to a significant weakness of this observer, namely, its assumed knowledge of the road surface conditions for accurate estimation of the lateral velocity. The solution presented relies on no additional measurements, such as engine or braking torque, thereby providing a solution for a sensor configuration found in conventional cars. Theoretical stability results are presented, subject to technical assumptions and a persistency-of-excitation condition.

\section{VehicLE Model}

\section{A. Rigid-Body Dynamics}

The vehicle is modeled as a rigid body and is studied in a body-fixed coordinate system with the origin located at the center of gravity, as illustrated in Fig. 1. Considering only the lateral direction, the equation of motion is

$$
\dot{v}_{y}=\frac{1}{m} \tau_{y}-r v_{x},
$$

where $v_{x}$ is the longitudinal velocity, $v_{y}$ is the lateral velocity, $r$ is the yaw rate, $\tau_{y}$ is the force acting in the lateral direction and $m$ is the vehicle mass. Using Newton's Second Law, we may write $(1 / m) \tau_{y}=a_{y}$, where $a_{y}$ is the lateral acceleration, to obtain the following equation (see [11]):

$$
\dot{v}_{y}=a_{y}-r v_{x} .
$$

All forces acting on the vehicle are disregarded, except those caused by friction between the road surface and the tires of the vehicle. These forces are denoted by the vectors $F_{i}=\left[\begin{array}{ll}F_{x, i} & F_{y, i}\end{array}\right]^{\top}, i=1, \ldots, 4$, with reference to wheel-fixed coordinate systems, which are rotated by angles $\delta_{i}$ with respect to the body-fixed coordinate system. We may therefore write

$$
\tau_{y}=\sum_{i=1}^{4}\left[\begin{array}{ll}
0 & 1
\end{array}\right] R\left(\delta_{i}\right) F_{i},
$$

where

$$
R\left(\delta_{i}\right)=\left[\begin{array}{cc}
\cos \delta_{i} & -\sin \delta_{i} \\
\sin \delta_{i} & \cos \delta_{i}
\end{array}\right]
$$




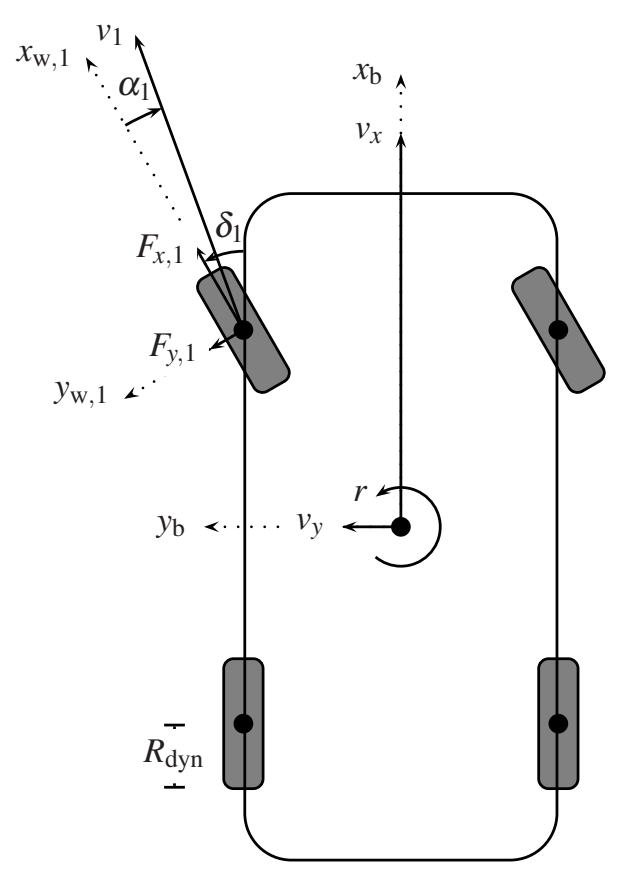

Fig. 1. Schematic overview of vehicle

are rotational matrices from the wheel-fixed coordinate systems to the body-fixed one.

\section{B. Friction Modeling}

The force vectors $F_{i}$ are modeled using a friction model. Several semi-empirical models for road-tire friction exist, the most well-known of which is often referred to as the Magic Formula (see [12]). In this paper, no particular friction model will be referred to. Rather, it will be assumed that certain structural properties hold for the friction model. ${ }^{1}$

Road-tire friction models are usually based on the normal force between the tires and the road, and tire slip values, which are measures of the relative difference between the vehicle velocity and the circumferential velocity of the tire. Exact definitions of slip vary, but one definition, which is the one referred to in this paper, is

$$
\lambda_{x, i}=\frac{\omega_{i} R_{\mathrm{dyn}}-\left\|v_{i}\right\| \cos \alpha_{i}}{\left\|v_{i}\right\|}, \quad \lambda_{y, i}=\sin \alpha_{i},
$$

where $\lambda_{x, i}$ and $\lambda_{y, i}$ are the slips in the longitudinal and lateral directions of tire number $i$, respectively; $R_{\mathrm{dyn}}$ is the dynamic radius of the tire; $\omega_{i}$ is the angular velocity of the wheel; $v_{i}$ is the velocity vector of the vehicle above the tire center; and $\alpha_{i}$ is the angle between the longitudinal direction of the tire and $v_{i}$.

It is common to define the friction coefficient $\mu=F / F_{z}$, where $F$ is the magnitude of the road-tire friction force and $F_{z}$ is the magnitude of the vertical force. In general, $\mu$ is lower on more slippery surfaces. However, $\mu$ is not a constant for a particular surface, so a more suitable value for describing

\footnotetext{
${ }^{1}$ A proprietary friction model is used in the experiments presented in Section V-B.
}

road surface conditions is the maximum road-tire friction coefficient, which is the maximum value of $\mu$ for a particular surface. We will assume that in the friction model, the difference in road-tire adhesion between particular surfaces is represented by the maximum road-tire friction coefficient, or a closely related value, denoted $\theta$. For simplicity, the value $\theta$ will be referred to as the friction parameter.

\section{OBSERVER}

\section{A. Notation}

We will estimate lateral velocity and the friction parameter, and we define a vector $x=\left[\begin{array}{ll}v_{y} & \theta\end{array}\right]^{\top}$ of these states. For some variable $z, \hat{z}$ represents its estimate and $\tilde{z}:=z-\hat{z}$. In the friction model, the forces are functions of the vector $x$ and several time-varying signals, so we write $\tau_{y}=\tau_{y}(t, x)$. As seen above, $a_{y}=(1 / m) \tau_{y}(t, x)$, and we also define $\hat{a}_{y}:=$ $(1 / m) \tau_{y}(t, \hat{x})$ (i.e., the lateral acceleration calculated based on estimated states), as well as $\tilde{a}_{y}:=a_{y}-\hat{a}_{y}$. We denote the closed ball with center at the origin and radius $z$ by $\bar{B}(z)$.

\section{B. Non-Adaptive Observer}

This paper is based on an observer from [10], which estimates longitudinal velocity, lateral velocity and yaw rate. It uses measurements of longitudinal acceleration, lateral acceleration, yaw rate, wheel angular velocities and steering wheel angle. The individual wheel angles $\delta_{i}$ can be calculated from the steering wheel angle. The measurements go into the friction model as time-varying signals for calculation of normal force and tire slip values. The estimate of the lateral velocity, given by

$$
\dot{\hat{v}}_{y}=a_{y}-r v_{x}-K_{v_{y}}\left(a_{y}-\hat{a}_{y}\right),
$$

is particularly sensitive to errors in the friction parameter because the feedback in this estimate is through the friction model. By contrast, the yaw rate is measured directly, and the longitudinal velocity estimation is based on feedback from the wheel angular velocities. This paper will therefore focus on the lateral velocity alone, and assume that $v_{x}$ and $r$ are known.

If the friction parameter is known, then, under a set of technical assumptions, the origin of the error dynamics resulting from the observer (3) is exponentially stable within a region. The goal in this paper is to find a parameter update law for estimation of the friction parameter which ensures accurate and globally asymptotically stable estimation of the lateral velocity when the actual friction parameter is unknown.

\section{Adaptive Observer}

In order to state some necessary assumptions, we define a vector $d$ as containing all time-varying signals upon which the calculation of friction forces depends, except $v_{y}$ and $\theta$, and write $F_{i}=F_{i}(d, x)$. It is assumed that $d$ includes the steering angles $\delta_{i}$.

Assumption 1: There exist compact sets $D_{d} \subset \mathbb{R}^{m}$ and $D_{x} \subset \mathbb{R}^{2}$ such that $(d, x) \in D_{d} \times D_{x} ; F_{i}(d, x),\left[\partial F_{i} / \partial x\right](d, x)$ and $\left[\partial^{2} F_{i} / \partial x^{2}\right](d, x)$ are continuous on $D_{d} \times \mathbb{R}^{2} ; d$ and $v_{y}$ are 
uniformly continuous in $t$ on $\mathbb{R}$; and there exist constants $c_{1}$, $c_{2}$ and $c_{3}$ such that for all $(t, x) \in \mathbb{R} \times \mathbb{R}^{2}$,

$$
\begin{gathered}
-c_{2} \leq \frac{1}{m} \frac{\partial \tau_{y}(t, x)}{\partial v_{y}} \leq-c_{1}<0, \\
\frac{1}{m}\left|\frac{\partial \tau_{y}(t, x)}{\partial \theta}\right| \leq c_{3} .
\end{gathered}
$$

Remark 1: Implicit in this assumption are some assumptions on the physical state of the vehicle in order to avoid singularities in the slip equations (2). Assumption 1 and Assumption 3 below are discussed in detail in Section IV.

Assumption 2: The friction parameter $\theta$ is constant, such that $\dot{\theta}=0$.

Assumption 3: The lateral force $\tau_{y}(t, x)$ can be written as a truncated Taylor series expansion, as follows:

$$
\tau_{y}(t, x)=\tau_{y}(t, \hat{x})+\frac{\partial \tau_{y}(t, \hat{x})}{\partial v_{y}} \tilde{v}_{y}+\frac{\partial \tau_{y}(t, \hat{x})}{\partial \theta} \tilde{\theta} .
$$

For ease of notation, we define

$$
\bar{\xi}_{v_{y}}(t, \hat{x}):=\frac{1}{m} \frac{\partial \tau_{y}(t, \hat{x})}{\partial v_{y}}, \quad \bar{\xi}_{\theta}(t, \hat{x}):=\frac{1}{m} \frac{\partial \tau_{y}(t, \hat{x})}{\partial \theta} .
$$

Subtracting $\tau_{y}(t, \hat{x})$ from both sides of (6) and dividing by $m$, we get the following expression for $\tilde{a}_{y}$ :

$$
\tilde{a}_{y}=\bar{\xi}_{v_{y}}(t, \hat{x}) \tilde{v}_{y}+\bar{\xi}_{\theta}(t, \hat{x}) \tilde{\theta} .
$$

Noting that $\hat{x}=x-\tilde{x}$, we also define $\xi_{v_{y}}(t, \tilde{x}):=\bar{\xi}_{v_{y}}(t, \hat{x})$ and $\xi_{\theta}(t, \tilde{x}):=\bar{\xi}_{\theta}(t, \hat{x})$ by considering $x$ a time-varying signal.

We propose the following observer:

$$
\begin{aligned}
\dot{\hat{v}}_{y} & =a_{y}-r v_{x}-K_{v_{y}}\left(a_{y}-\hat{a}_{y}\right), \\
\dot{\hat{\theta}} & =-\Gamma \bar{\xi}_{\theta}(t, \hat{x}) \bar{\xi}_{v_{y}}^{-1}(t, \hat{x})\left(a_{y}-\hat{a}_{y}\right),
\end{aligned}
$$

with tuning gains $K_{v_{y}}>0$ and $\Gamma>0$. Note that $(8 \mathrm{~b})$ is well-defined, because, according to Assumption $1, \bar{\xi}_{v_{y}}(t, \hat{x}) \leq$ $-c_{1}<0$. In the following section, the stability properties of this observer will be investigated.

\section{Stability}

Subtracting (8a) from (1), we obtain the following error dynamics:

$$
\begin{aligned}
\dot{\tilde{v}_{y}} & =K_{v_{y}} \tilde{a}_{y}, \\
\dot{\tilde{\theta}} & =\Gamma \xi_{\theta}(t, \tilde{x}) \xi_{v_{y}}^{-1}(t, \tilde{x}) \tilde{a}_{y} .
\end{aligned}
$$

Note that Assumptions 1-2 imply that the right-hand side of (9) is continuous in $t$ and locally Lipschitz continuous in $\tilde{x}$, uniformly in $t$.

Lemma 1: If Assumptions 1-3 hold, then the origin of (9) is uniformly globally stable (UGS).

Proof: Define a Lyapunov function candidate $V: \mathbb{R}^{2} \rightarrow$ $\mathbb{R}$ as $V(\tilde{x}):=\frac{1}{2}\left(\tilde{v}_{y}^{2}+K_{v_{y}} \Gamma^{-1} \tilde{\theta}^{2}\right)$. Its time derivative along the trajectories of (9) is

$$
\dot{V}(t, \tilde{x})=K_{v_{y}} \tilde{a}_{y} \tilde{v}_{y}+K_{v_{y}} \xi_{\theta}(t, \tilde{x}) \xi_{v_{y}}^{-1}(t, \tilde{x}) \tilde{a}_{y} \tilde{\theta} .
$$

Using (7) to substitute for $\tilde{v}_{y}$, we get that

$$
\dot{V}(t, \tilde{x})=K_{v_{y}} \xi_{v_{y}}^{-1}(t, \tilde{x}) \tilde{a}_{y}^{2} \leq-\frac{K_{v_{y}}}{c_{2}} \tilde{a}_{y}^{2} \leq 0 .
$$

Because $V$ is radially unbounded and $\dot{V}$ is negative semidefinite, it follows that the origin of (9) is UGS (see [13, Def. $1])$.

The time derivative of the Lyapunov function is not negative definite, so we cannot directly conclude with asymptotic stability. If we assume that $\tilde{a}_{y}$ is uniformly continuous, it is possible to invoke Barbălat's Lemma (see [14], [15]) to conclude that $\lim _{t \rightarrow \infty} \tilde{a}_{y}=0$. Inspecting (7), one might intuitively think that if $\bar{\xi}_{v_{y}}$ and $\bar{\xi}_{\theta}$ vary independently with time, this cannot happen unless $\tilde{v}_{y}$ and $\tilde{\theta}$ also go to zero. In the following theorem, a formal condition is given for uniform global asymptotic stability (UGAS) of the origin of the error dynamics.

Theorem 1: Suppose that for each $\chi \in \mathbb{R}^{2} \backslash\{0\}$, there exist $\varepsilon>0$ and $T>0$ such that $\forall t \in \mathbb{R}$,

$$
\begin{aligned}
\int_{t}^{t+T} \xi_{v_{y}}^{2}(\tau, \chi) \mathrm{d} \tau \int_{t}^{t+T} \xi_{\theta}^{2}(\tau, \chi) \mathrm{d} \tau \\
\geq\left(\int_{t}^{t+T} \xi_{v_{y}}(\tau, \chi) \xi_{\theta}(\tau, \chi) \mathrm{d} \tau\right)^{2}+\varepsilon .
\end{aligned}
$$

If Assumptions 1-3 hold, then the origin of (9) is UGAS.

Proof: For this proof, [13, Cor. 3], which is a reformulation of [16, Th. 6.3], will be utilized. We may write $\dot{\tilde{x}}=f(t, \tilde{x})=A(t, \tilde{x}) \tilde{x}$, where

$$
A(t, \tilde{x}):=\left[\begin{array}{cc}
K_{v_{y}} \xi_{v_{y}}(t, \tilde{x}) & K_{v_{y}} \xi_{\theta}(t, \tilde{x}) \\
\Gamma \xi_{\theta}(t, \tilde{x}) & \Gamma \xi_{\theta}^{2}(t, \tilde{x}) \xi_{v_{y}}^{-1}(t, \tilde{x})
\end{array}\right] .
$$

From Lemma 1, the origin of this system is UGS. Both $V$ and $f$ are bounded for bounded $\tilde{x}$, and hence for each $\Delta>0$, there exists a number $\varepsilon_{1}>0$ such that $\forall(t, \tilde{x}) \in \mathbb{R} \times \bar{B}(\Delta)$, $\max \{|V(\tilde{x})|,\|f(t, \tilde{x})\|\} \leq \varepsilon_{1}$. Define $Y: \mathbb{R}^{2} \times \mathbb{R}^{2} \rightarrow \mathbb{R}$ as $Y(z, \psi):=-C \psi^{\top} \psi$, where $C=K_{v_{y}} c_{1}^{2} /\left(K_{v_{y}}^{2} c_{1}^{2} c_{2}+\Gamma^{2} c_{2} c_{3}^{2}\right)$. Then

$$
Y(\tilde{x}, f(t, \tilde{x}))=-C\left(K_{v_{y}}^{2}+\Gamma^{2} \xi_{\theta}^{2}(t, \tilde{x}) \xi_{v_{y}}^{-2}(t, \tilde{x})\right) \tilde{a}_{y}^{2} .
$$

It follows from (10) and the bounds on $\xi_{v_{y}}(t, \tilde{x})$ and $\xi_{\theta}(t, \tilde{x})$ that $\dot{V}(t, \tilde{x}) \leq Y(\tilde{x}, f(t, \tilde{x}))$. Because $Y(z, \psi)$ is negative semidefinite, it follows that $(z, \psi) \in \bar{B}(\Delta) \times \bar{B}\left(\varepsilon_{1}\right) \Longrightarrow$ $Y(z, \psi) \leq 0$. From the definition of $Y$, it is easy to see that

$$
(z, \psi) \in \bar{B}(\Delta) \times \bar{B}\left(\varepsilon_{1}\right), Y(z, \psi)=0 \Longrightarrow \psi=0 .
$$

Thus, the origin of (9) is UGAS if $f$ is uniformly $\delta$ persistently exciting ( $\delta$-PE) with respect to $\tilde{x}$, as defined in [13]. To show when this is the case, [13, Lemma 1] is used. Consider the matrix $Q(t)=\int_{t}^{t+T} A^{\top}(\tau, \chi) A(\tau, \chi) \mathrm{d} \tau$. Writing out the integral yields

$$
\begin{aligned}
& Q(t)=K_{v_{y}}^{2} \int_{t}^{t+T}\left[\begin{array}{cc}
\xi_{v_{y}}^{2}(\tau, \chi) & \xi_{v_{y}}(\tau, \chi) \xi_{\theta}(\tau, \chi) \\
\xi_{v_{y}}(\tau, \chi) \xi_{\theta}(\tau, \chi) & \xi_{\theta}^{2}(\tau, \chi)
\end{array}\right] \mathrm{d} \tau \\
& +\Gamma^{2} \int_{t}^{t+T}\left[\begin{array}{cc}
\xi_{\theta}^{2}(\tau, \chi) & \xi_{\theta}^{3}(\tau, \chi) \xi_{v_{y}}^{-1}(\tau, \chi) \\
\xi_{\theta}^{3}(\tau, \chi) \xi_{v_{y}}^{-1}(\tau, \chi) & \xi_{\theta}^{4}(\tau, \chi) \xi_{v_{y}}^{-2}(\tau, \chi)
\end{array}\right] \mathrm{d} \tau .
\end{aligned}
$$

Note that both terms are positive semidefinite, and consider the first term, denoted $Q_{1}(t)$. Its first-order principal minor is $K_{v_{y}}^{2} \int_{t}^{t+T} \xi_{v_{y}}^{2}(\tau, \chi) \mathrm{d} \tau \geq K_{v_{y}}^{2} c_{1}^{2} T>0$. Its second-order principal 
minor, or determinant, is

$$
\begin{aligned}
K_{v_{y}}^{2} \int_{t}^{t+T} \xi_{v_{y}}^{2}(\tau, \chi) \mathrm{d} \tau \int_{t}^{t+T} \xi_{\theta}^{2}(\tau, \chi) \mathrm{d} \tau \\
-K_{v_{y}}^{2}\left(\int_{t}^{t+T} \xi_{v_{y}}(\tau, \chi) \xi_{\theta}(\tau, \chi) \mathrm{d} \tau\right)^{2} .
\end{aligned}
$$

The condition (11) therefore implies positive definiteness of $Q_{1}(t)$. It also implies that $Q_{1}(t)$ has a uniformly lower bounded determinant, which together with the fact that $Q_{1}(t)$ has uniformly bounded elements means that its minimum eigenvalue is also uniformly lower bounded by some value $\varepsilon_{2}>0$. We can therefore write $Q(t) \geq Q_{1}(t) \geq \varepsilon_{2} I$.

This implies that for each $\chi \neq 0$ there exist $\varepsilon_{3}>0$ and $T>0$ such that $\forall t \in \mathbb{R}, \chi^{\top} Q(t) \chi=\int_{t}^{t+T}\|f(\tau, \chi)\|^{2} \mathrm{~d} \tau \geq \varepsilon_{3}$. Boundedness of the integrand for each $\chi$ in turn implies that for each $\chi \neq 0$ there exist $\varepsilon_{4}>0$ and $T>0$ such that $\forall t \in \mathbb{R}$, $\int_{t}^{t+T}\|f(\tau, \chi)\| \mathrm{d} \tau \geq \varepsilon_{4}$, which means that $f$ is $\mathrm{U} \delta$-PE with respect to $\tilde{x}$.

Remark 2: According to the Cauchy-Schwartz Inequality, the first term of (12) is equal to or larger than the second term, and they are equal only if the signals $\xi_{v_{y}}(\tau, \chi)$ and $\xi_{\theta}(\tau, \chi)$ are linearly dependent (see [17, Th. 1.9]). Hence, (11) is a requirement of linear independence between $\xi_{v_{y}}(t, \tilde{x})$ and $\xi_{\theta}(t, \tilde{x})$ for fixed, nonzero $\tilde{x}$.

There is an intuitive interpretation of the condition for stability stated by Theorem 1 . The functions $\xi_{v_{y}}$ and $\xi_{\theta}$ are nonlinear functions which depend on several time-varying signals. Because $\xi_{v_{y}}$ and $\xi_{\theta}$ are substantially different from each other, they will behave differently when excited by these time-varying signals. Therefore, if there is some variation in these signals in the interval between $t$ and $t+T, \xi_{v_{y}}(t, \chi)$ and $\xi_{\theta}(t, \chi)$ will not be linearly dependent for fixed $\chi$ over this interval. If, on the other hand, all the time-varying signals are kept constant, the functions $\xi_{v_{y}}(t, \chi)$ and $\xi_{\theta}(t, \chi)$ will themselves be constant and, therefore, linearly dependent.

In practical terms, this leads to a requirement of a somewhat varied driving pattern. Rather than driving in a straight line or a circle at constant speed, UGAS requires a certain amount of turning, acceleration or braking. Maneuvers causing large variation in the lateral tire slip values are particularly useful. It is not required that this happen all the time, but there must exist a $T$, arbitrarily large, such that within any time interval of length $T$, there is sufficient variation to excite the system.

If the condition does not hold because the vehicle is driven along a straight path for an indefinite amount of time, we can state a separate result which does hold in this case.

Theorem 2: Suppose that Assumptions 1-3 hold and that for each $\varepsilon_{1}>0$ there exists a $\varepsilon_{2}>0$ such that $\left|\hat{v}_{y}\right| \geq \varepsilon_{1}, r=0$, $\delta=0 \Longrightarrow\left|\hat{a}_{y}\right| \geq \varepsilon_{2}$. If $\forall t \geq t_{0}, a_{y}=0, v_{y}=0, r=0, \delta=0$, then $\lim _{t \rightarrow \infty} \tilde{v}_{y}=0$.

Proof (Outline): Using the Lyapunov function from Lemma 1, Barbălat's Lemma can be used to conclude that $\lim _{t \rightarrow \infty} \hat{a}_{y}=0$, which, by the extra condition in Theorem 2 , implies that $\lim _{t \rightarrow \infty} \hat{v}_{y}=0$.

Remark 3: The practical meaning of the extra condition in Theorem 2 is that if the steering wheel angle is zero and the vehicle is not rotating, any nonzero lateral velocity will generate a nonzero lateral acceleration. This is reasonable from a physical point of view, and is likely to hold for most friction models, at least if the possibility of a friction-less surface is excluded. The problem of physically unreasonable friction parameter estimates is addressed in the next section.

\section{E. Robustness Modification}

It has been assumed up to this point that Assumption 1 holds for all $\theta \in \mathbb{R}$, but from physical considerations, $\theta$ is confined to a small region. In particular, we can assume that on any surface, some friction forces are generated, and that the friction parameter is upper bounded. Inserting physically unreasonable values into a friction model may result in violation of the assumptions, for example by causing a singularity or switching the sign of (4), and it is therefore preferable if $\hat{\theta}$ remains within a physically reasonable region.

This type of problem can be handled by using a projection scheme to prevent the trajectories of the system from leaving some convex valid region (see [18]). The parameter update law is therefore altered as described in the next theorem.

Theorem 3: Suppose that there are known bounds $\theta_{\min }$ and $\theta_{\max }$ on the friction parameter, such that $0<\theta_{\min } \leq \theta \leq$ $\theta_{\max }$, and that the parameter update law (8b) is substituted with

$$
\begin{gathered}
\dot{\hat{\theta}}=(\operatorname{sat}(\rho(t, \hat{x}))-1) \Gamma \bar{\xi}_{\theta}(t, \hat{x}) \bar{\xi}_{v_{y}}^{-1}(t, \hat{x}) \tilde{a}_{y}, \\
\rho(t, \hat{x})= \begin{cases}\left(\theta_{\min }-\hat{\theta}\right) / \theta_{\varepsilon}, & \hat{\theta} \leq \theta_{\min }, \bar{\xi}_{\theta}(t, \hat{x}) \tilde{a}_{y}<0, \\
\left(\hat{\theta}-\theta_{\max }\right) / \theta_{\varepsilon}, & \hat{\theta} \geq \theta_{\max }, \bar{\xi}_{\theta}(t, \hat{x}) \tilde{a}_{y}>0, \\
0, & \text { otherwise, }\end{cases}
\end{gathered}
$$

where $\theta_{\varepsilon}$ is some arbitrarily small, positive value and sat $(\cdot)$ is a saturation with lower limit 0 and upper limit 1 . Then, under the same conditions as in Theorem 1, the origin of the observer error dynamics is UGAS, and if $\theta_{\min }-\theta_{\varepsilon} \leq \hat{\theta} \leq$ $\theta_{\max }+\theta_{\varepsilon}$ at time $t_{0}$, then for all $t \geq t_{0}, \theta_{\min }-\theta_{\varepsilon} \leq \hat{\theta} \leq$ $\theta_{\max }+\theta_{\varepsilon}$.

Proof (Outline): Inspection of (13) reveals that the modification to the parameter update law adds a non-positive term to the time derivative of the Lyapunov function from Lemma 1. Part one of Theorem 3 then follows from including this perturbation in the proofs of Lemma 1 and Theorem 1. Part two of Theorem 3 follows from continuity of the solutions and the fact that for $\hat{\theta}=\theta_{\min }-\theta_{\varepsilon}, \dot{\hat{\theta}} \geq 0$, and for $\hat{\theta}=\theta_{\max }+\theta_{\varepsilon}, \dot{\hat{\theta}} \leq 0$.

\section{Discussion of Assumptions}

\section{A. Assumption 1}

In Assumption 1, certain assumptions are made on the continuity of the friction model, and the partial derivatives of $\tau_{y}$ with respect to $v_{y}$ and $\theta$ are assumed to be globally bounded. The validity of these assumptions depends on the friction model used, but clearly we cannot tolerate singularities in the model. Considering the definition of longitudinal tire slip in Section II-B, it is easy to see that the expression can become singular at low speeds. In [10], the vehicle 
is assumed to always be forward-moving, and the stability results are regional, such that the friction model can never become singular. When estimating only $v_{y}$, and not $v_{x}$ and $r$, we can avoid singularity of the friction model if we assume that the vehicle is moving forward with a certain velocity and is not rotating unreasonably fast. This is therefore implied by Assumption 1 .

As discussed previously, it is unlikely that the assumptions hold for all friction parameters, but this problem is circumvented by the method described in Section III-E. It is also possible that problems may occur if the magnitude of the lateral velocity estimate attains unreasonably large values. If this is the case, the same method may be used to restrict $\hat{v}_{y}$ to physically reasonable values.

The partial derivative of $\tau_{y}$ with respect to $v_{y}$ is assumed strictly negative. This is also assumed in [10] and forms the basis of the original, non-adaptive observer. The same arguments as in [10] therefore largely apply. There is a difference, however, in that we assume that this holds for arbitrarily large lateral velocities, which may cause very large slip angles $\alpha_{i}$, and this may not be realistic. Again, the same method as the one described in Section III-E may be employed in order to restrict the magnitude of $\hat{v}_{y}$, so the global assumption is not significantly stricter than the regional one.

\section{B. Assumption 3}

According to the Mean Value Theorem, (6) would hold exactly if the partial derivatives were taken at some point between the real and the estimated values of $v_{y}$ and $\theta$. Instead, we assume that it holds when they are taken at the estimated values. This obviously does not hold in general, but it holds approximately for small estimation errors, and the error committed vanishes at the origin of the error dynamics.

As stated in [13], UGAS equilibrium points are locally input-to-state stable and therefore robust with respect to small perturbations, and we may therefore expect that, at least locally, the approximations made will not cause problems. This is verified experimentally in the next section.

It may also be pointed out that the stability analysis is fully nonlinear and that Assumption 3 is only needed in order to evaluate the factors in (7) from known quantities, for implementation of the parameter update law.

\section{Practical Issues and Experimental Results}

The observer has been implemented and tested on recorded measurements from a car, using $K_{v_{y}}=1.0, \Gamma=0.7, \theta_{\min }=$ $0.1, \theta_{\max }=1.0$ and $\theta_{\varepsilon}=0.01$. A slight modification was made to the observer in order to improve results. This is described in the next section.

\section{A. Modification for Low Excitation}

In the theoretical analysis, it was found that convergence of the observer relies on a certain amount of variation in the driving pattern. This is clearly reflected in the experimental results. In particular, the quality of the friction parameter estimate tends to be poor when there is insufficient variation in the lateral acceleration, and therefore little information in $\tilde{a}_{y}$, and it may in fact drift off. Simulations show that using an estimate of the friction parameter that is much too low may lead to significantly reduced observer performance, and is far worse than using one that is too high. This means that problems may arise if a lack of variation leads the friction parameter to drift toward artificially low values.

The practical solution chosen is to let $\hat{\theta}$ be attracted toward what we may term a safe value $\theta_{s}$ whenever the lateral acceleration error $\tilde{a}_{y}$ remains close to zero. In practice, this is implemented by adding a term $K_{s} l\left(\bar{a}_{y}^{2}\right)\left(\theta_{s}-\hat{\theta}\right)$ to the parameter update law (13), where $l\left(\bar{a}_{y}^{2}\right)$ is a logical expression evaluating to 1 if $\bar{a}_{y}^{2}<b$ and 0 otherwise. The value $\bar{a}_{y}^{2}$ is a low-pass filtered version of $\tilde{a}_{y}^{2}$, given by its Laplace transform as $\bar{a}_{y}^{2}(s)=1 /\left(T_{s} s+1\right) \tilde{a}_{y}^{2}(s)$, and $b$ is some threshold value. In the experiments, the values $K_{s}=0.1$, $b=0.2 \theta_{s}=1.0$ and $T_{s}=2.0$ were used.

A too high value for $\hat{\theta}$ generally results in a lower magnitude of $\hat{v}_{y}$. However, a lack of excitation often goes together with a low magnitude of $v_{y}$, and it is safer to estimate the magnitude of $v_{y}$ too low than too high, as explained in [2]. Moreover, $\hat{\theta}$ tends to quickly come down to low values when there is more excitation.

\section{B. Experimental Results}

In this section, results are shown for particular test cases. The measurements of lateral acceleration, yaw rate, steering wheel angle and wheel angular velocities are provided by the test vehicle's electronic stability program (ESP) unit; the longitudinal acceleration is measured with a separate inertial measurement unit (IMU); and the longitudinal and lateral velocities are measured, for validation purposes only, using optical correlation sensors mounted on the test vehicle. In the tests, longitudinal velocity estimated using the modular design from [10] is used as input to the lateral velocity observer, in order to give a realistic impression of performance. Replacing the estimate by the optical measurement does not alter the results significantly for the tests presented.

1) Slalom Maneuver on High-Friction Surface: The first test is a slalom maneuver on a high-friction surface, where the initial value of the friction parameter is set low, at 0.1 . For about $12 \mathrm{~s}$, the longitudinal velocity remains around $27 \mathrm{~m} / \mathrm{s}$. It then decreases to about $13 \mathrm{~m} / \mathrm{s}$ at $t=22 \mathrm{~s}$, and abruptly returns to around $27 \mathrm{~m} / \mathrm{s}$. The results can be seen in Fig. 2 . It is clear that $\hat{\theta}$ climbs and is stopped only by the upper limit on the friction parameter. The observer overall correctly estimates the velocity and a high friction parameter.

2) Circle Maneuver on Low-Friction Surface: The second test is a circle maneuver on ice. The real $\theta$ probably varies, whereas the initial value for $\hat{\theta}$ is set high, at 1.0 . The longitudinal velocity increases approximately from $16 \mathrm{~m} / \mathrm{s}$ to $24 \mathrm{~m} / \mathrm{s}$ at $t=14 \mathrm{~s}$. It then decreases to about $16 \mathrm{~m} / \mathrm{s}$ at $t=17 \mathrm{~s}$, and increases slightly again from there. The results can be seen in Fig. 3. This experiment clearly shows how $\hat{\theta}$ drops to appropriate, low values as soon as there is enough variation in the lateral direction, and it is therefore an illustration of the $\mathrm{U} \delta$-PE condition from the theoretical 


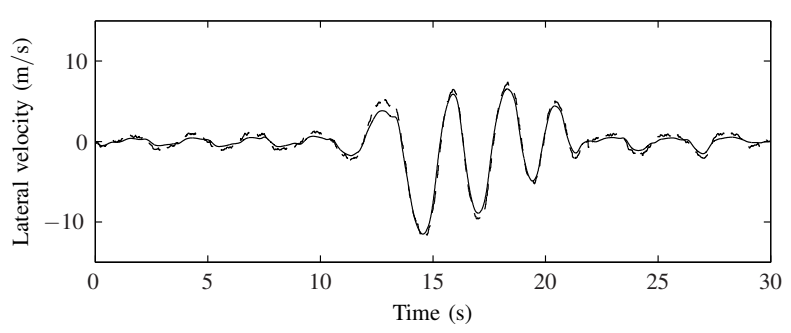

(a) Real (dashed) and estimated (solid) lateral velocity

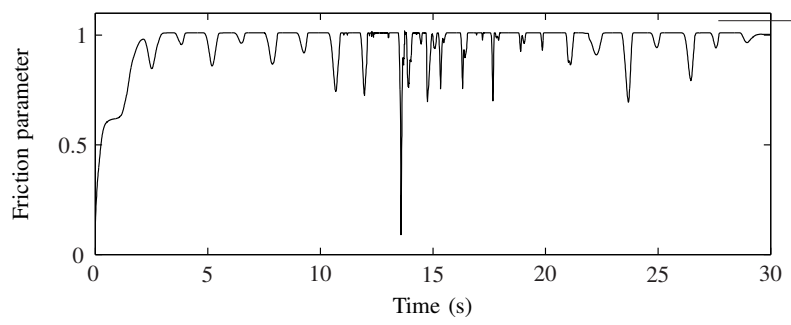

(b) Estimated friction parameter

Fig. 2. Slalom maneuver

analysis. The estimate $\hat{v}_{y}$ tracks $v_{y}$ quite well. Note that the rise in $\hat{\theta}$ at around $t=17 \mathrm{~s}$ is likely attributable to an actual change in the road surface conditions during the physical test, as the vehicle may slide off the test track after the driver loses control around $t=15 \mathrm{~s}$.

\section{Discussion}

The observer has been tested successfully on more test cases than the ones discussed above. It is primarily effective at giving an accurate estimate of the lateral velocity, with the estimated friction parameter tending to take on appropriate values whenever there is enough variation in the lateral direction. The estimate $\hat{\theta}$ will tend to capture the effects of other, unmodeled phenomenons as well, and it can therefore not be expected to represent the road surface conditions accurately. This, together with the fact that the road surface conditions can change during regular driving, means that the assumption of a constant friction parameter should be seen as an approximation. It seems likely, however, that-perhaps with some post-processing of the estimate-the adapted friction parameter estimate may provide useful information about the road surface conditions.

\section{CONCLUding Remarks}

An adaptive observer has been presented with the aim of improving an estimate of the lateral velocity when the road surface conditions are unknown. According to the theoretical analysis, UGAS of the origin of the observer error dynamics relies on a condition essentially requiring a varied driving pattern. Experimental results support this and show that the lateral velocity estimate remains accurate even when the initial estimate of the friction parameter is completely wrong.

\section{ACKNOWLEDGEMENTS}

The authors wish to acknowledge the contribution of Dr. Antonio Loría, who gave of his time for helpful discussion regarding Theorem 1.

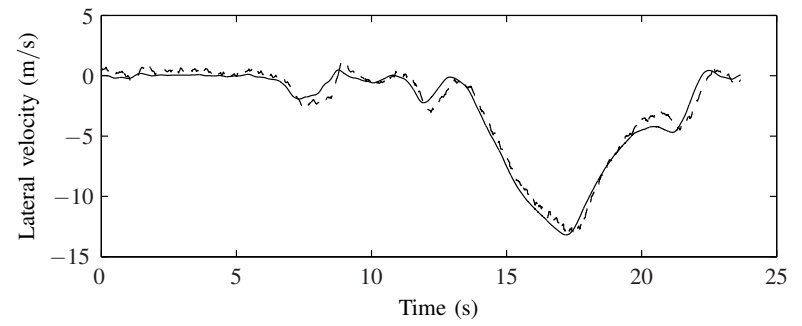

(a) Real (dashed) and estimated (solid) lateral velocity

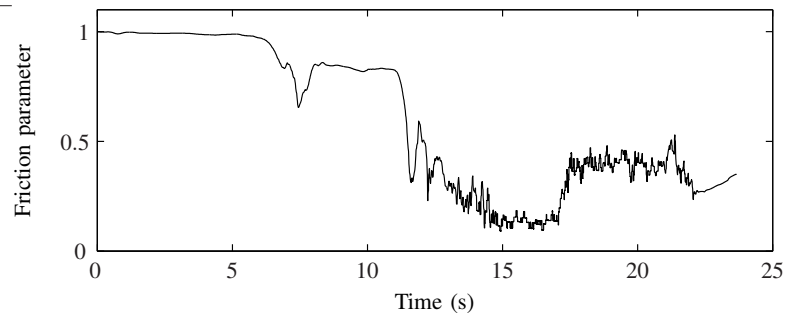

(b) Estimated friction parameter

Fig. 3. Circle maneuver on ice

\section{REFERENCES}

[1] E. Ono, K. Asano, M. Sugai, S. Ito, M. Yamamoto, M. Sawada, and Y. Yasui, "Estimation of automotive tire force characteristics using wheel velocity," Contr. Eng. Pract., vol. 11, pp. 1361-1370, 2003.

[2] Y. Fukada, "Slip-angle estimation for vehicle stability control," Vehicle System Dynamics, vol. 32, pp. 375-388, 1999.

[3] A. Hac and M. D. Simpson, "Estimation of vehicle side slip angle and yaw rate," in Proc. SAE World Congress, Detroit, MI, 2000.

[4] F. Gustafsson, "Slip-based tire-road friction estimation," Automatica, vol. 33, no. 6, pp. 1087-1099, 1997.

[5] L. R. Ray, "Nonlinear tire force estimation and road friction identification: Simulation and experiments," Automatica, vol. 33, no. 10, pp. 1819-1833, 1997.

[6] A. Suissa, Z. Zomotor, and F. Böttiger, "Method for determining variables characterizing vehicle handling," US patent 5557520, 1996.

[7] M. C. Best, T. J. Gordon, and P. J. Dixon, "An extended adaptive Kalman filter for real-time state estimation of vehicle handling dynamics," Vehicle System Dynamics, vol. 34, pp. 57-75, 2000.

[8] H. Nishira, T. Kawabe, and S. Shin, "Road friction estimation using adaptive observer with periodical $\sigma$-modification," in Proc. IEEE Int. Conf. Contr. Appl., Kohala Coast, HI, 1999.

[9] C. Canudas-de-Wit, M. L. Petersen, and A. Shiriaev, "A new nonlinear observer for tire/road distributed contact friction," in Proc. 42nd IEEE Conf. Dec. Contr., Maui, HI, 2003.

[10] L. Imsland, T. A. Johansen, T. I. Fossen, H. F. Grip, J. C. Kalkkuhl, and A. Suissa, "Vehicle velocity estimation using nonlinear observers," Automatica, vol. 42, no. 12, 2006.

[11] U. Kiencke and L. Nielsen, Automotive Control Systems. Springer, 2000.

[12] H. B. Pacejka, Tyre and Vehicle Dynamics. Butterworth-Heinemann, 2002.

[13] A. Loría, E. Panteley, D. Popović, and A. R. Teel, "A nested Matrosov theorem and persistency of excitation for uniform convergence in stable nonautonomous systems," IEEE Trans. Automat. Contr., vol. 50, no. 2, pp. 183-198, 2005.

[14] I. Barbălat, "Systèmes d'équations différentielles d'oscillations non linéaires," Rev. Math. Pures Appl., vol. 4, no. 2, pp. 267-270, 1959.

[15] H. K. Khalil, Nonlinear Systems, 3rd ed. Upper Saddle River, NJ: Prentice Hall, 2002.

[16] Z. Artstein, "Uniform asymptotic stability via the limiting equations," J. Differ. Equat., vol. 27, no. 2, pp. 172-189, 1978.

[17] N. Young, An introduction to Hilbert space. Cambridge, United Kingdom: Cambridge University Press, 1988.

[18] P. A. Ioannou and J. Sun, Robust Adaptive Control. Upper Saddle River, NJ: Prentice Hall, 1996. 